\title{
Searches for sterile neutrinos at the DANSS experiment
}

\author{
I. Alekseev, A. Kobyakin, I. Machikhiliyan, V. Nesterov, N. Pogorelov, V. Rusinov, \\ E. Samigullin, N. Skrobova, A. Starostin, D. Svirida*, E. Tarkovsky \\ Alikhanov Institute for Theoretical and Experimental Physics NRC 'Kurchatov Institute', \\ Moscow, Russia \\ ${ }^{*}$ E-mail: Dmitry.Svirida@itep.ru
}

V. Belov, V. Brudanin, V. Egorov, D. Filosofov, M. Fomina, Z. Hons, S. Kazartcev, A. Kuznetsov, D. Medvedev, A. Olshevsky, D. Ponomarev, I. Rozova, N. Rumyantseva, Ye. Shevchik, M. Shirchenko, Yu. Shitov, J. Vlasek, I. Zhitnikov, D. Zinatulina

Joint Institute for Nuclear Research, Dubna, Moscow region, Russia

\section{Danilov}

Lebedev Physical Institute of the Russian Academy of Sciences, Moscow, Russia

DANSS is a one cubic meter highly segmented solid scintillator detector. It consists of 2500 scintillator strips $\left(100 \times 4 \times 1 \mathrm{~cm}^{3}\right)$, covered with gadolinium loaded reflective coating and read out with SiPMs via wave length shifting fibers. Groups of 50 strips are also read out by conventional PMTs. DANSS is placed under a $3 \mathrm{GW}$ industrial reactor at the Kalinin NPP (Russia) on a movable platform. The distance from the reactor core center can be changed from $10.7 \mathrm{~m}$ to $12.7 \mathrm{~m}$. The reactor building provides about $50 \mathrm{~m}$.w.e. shielding against cosmic backgrounds, which reduces the background drastically. The inverse beta decay (IBD) process is used to detect antineutrinos. DANSS detects about 5000 IBD events per day with the background from cosmic muons of about $3 \%$. The dependence of the antineutrino spectrum on the fuel composition is clearly observed. Sterile neutrinos are searched for assuming a 4 neutrino model (3 active and 1 sterile neutrino). The exclusion area in the sterile neutrino parameter plane is obtained using a ratio of positron energy spectra collected at different distances. Therefore, the results do not depend on the shape and normalization of the reactor antineutrino spectrum, as well as on the detector efficiency. Results are based on 1.088 million antineutrino events. The excluded area covers a wide range of the sterile neutrino parameters up to $\sin ^{2} 2 \theta_{14}<0.01$ in the most sensitive region. The Reactor Antineutrino Anomaly optimum point is excluded at the confidence level higher than $5 \sigma$.

Neutrino Oscillation Workshop (NOW2018)

9 - 16 September, 2018

Rosa Marina (Ostuni, Brindisi, Italy)

\footnotetext{
* Speaker.
} 
The DANSS detector [1] is designed to fully comply with the strictest reactor safety requirements. The detector position right below an industrial pressurized water reactor combines the advantage of the highest antineutrino flux of about $5 \cdot 10^{13} \mathrm{~cm}^{-2} \mathrm{~s}^{-1}$ with that of the 50 m.w.e. overburden due to the amount of matter from the reactor body and shielding. The inverse beta decay is detected, as commonly spread, as a pair of time correlated events composed of positron and neutron candidates. Fine detector segmentation helps much with the background suppression based on spatial information and allows the purification of the positron energy from the annihilation photons. Multilayer passive shielding of the detector provides the reduction of the ambient thermal neutron and gamma backgrounds by three orders of magnitude. Yet the accidental background rate is comparable to that of the IBD process, but the procedure of its subtraction is strict and does not increase the statistical error.

Cosmic muons can initiate processes that exactly mimic the IBD reaction. Active veto system allows to tag approximately $95 \%$ of muon-induced events and build corresponding muon-tagged distributions. The exact fraction of muon events that penetrate untagged through the veto system can be determined from the 'reactor OFF' data, where the majority of the events have cosmic origin. Figure 1 presents the comparison of the 'reactor OFF' data to the muon-tagged spectrum, which is scaled to the same level. In the data at full reactor power the cosmic background is subtracted on the position-dependent basis as a muon-tagged spectrum scaled down by the veto transparency. The dashed line in fig. 1 shows the background from the fast neutrons, born mostly in the surrounding concrete. Their spectrum is linearly extrapolated from the energy region of $10-16 \mathrm{MeV}$ and is subtracted separately.

Due to the advantages of the detector location and because of the elaborate data analysis procedure DANSS features unprecedented antineutrino counting rate of about 5000 per day in the position closest to the reactor core. At the same time the muon background amounts to only 130

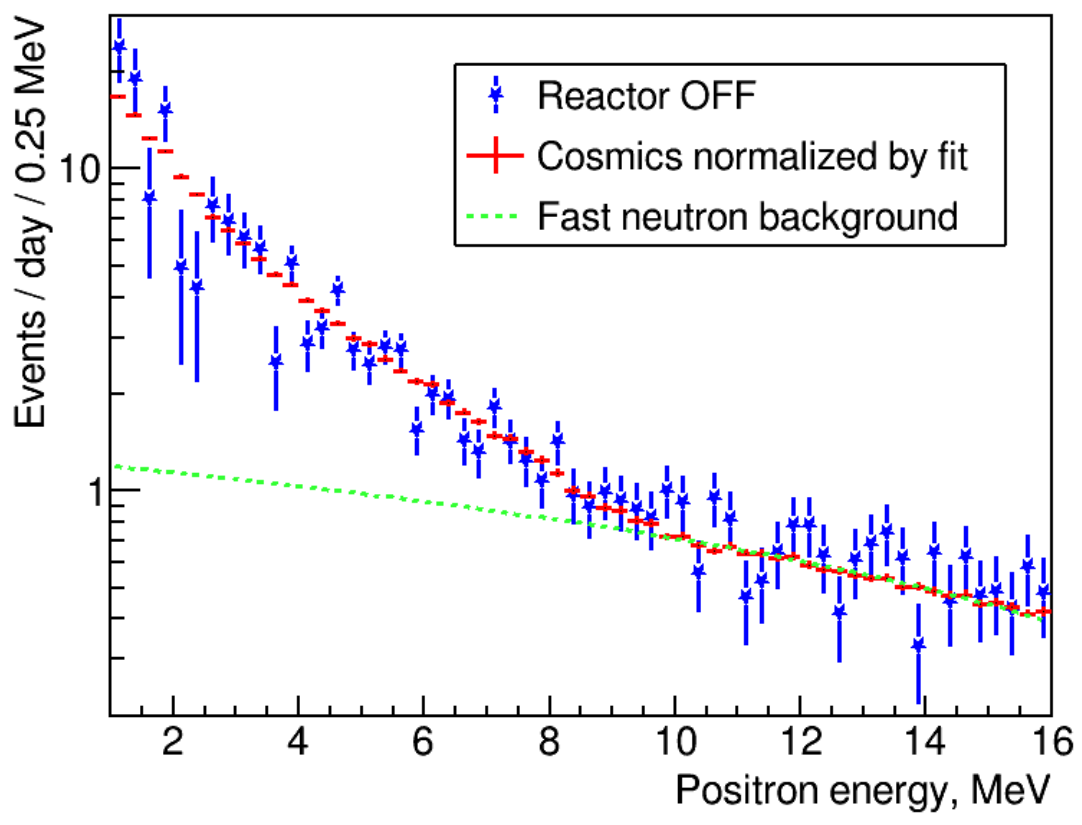

Figure 1: Reactor OFF positron spectrum in comparison to the cosmic background, scaled to the same level. 


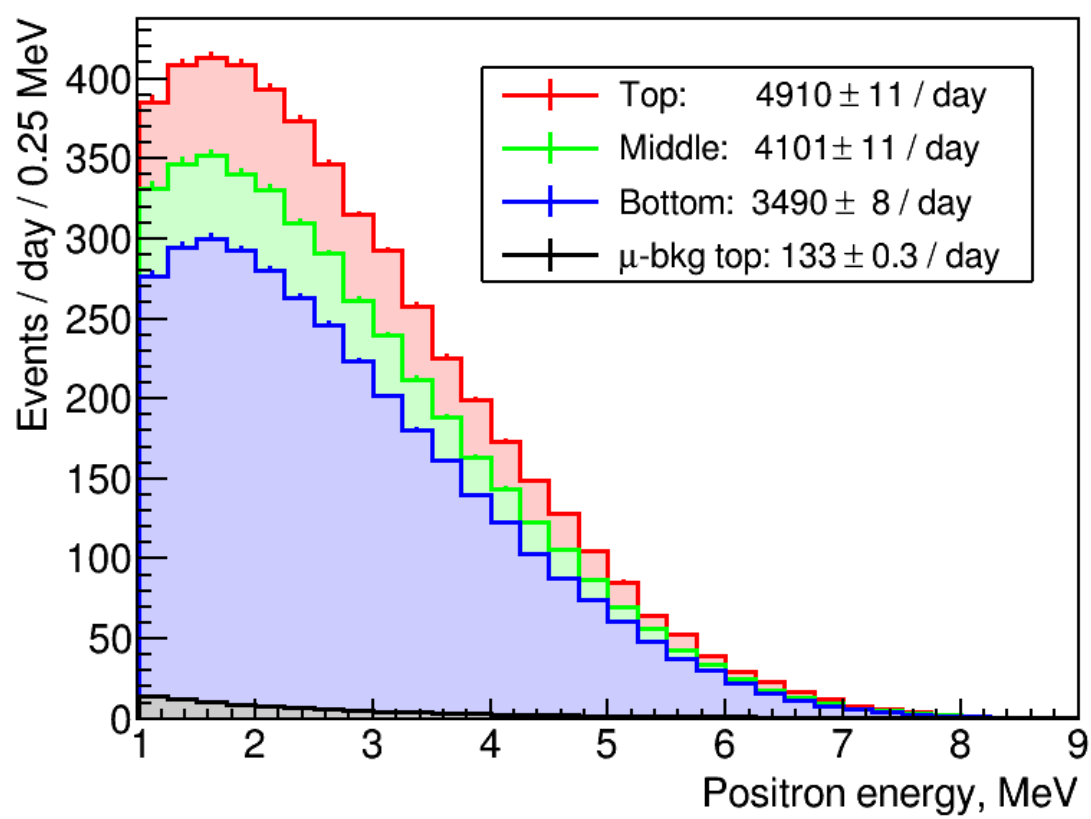

Figure 2: Positron spectra at the three detector positions.

events per day and gets accurately subtracted. The positron spectra obtained in the three detector positions are shown in fig. 2 together with the scaled spectrum of the cosmic background.

The idea of sterile neutrino searches with DANSS is based on the possibility to measure antineutrino spectrum dependent on the distance from their source with one and the same detector. Data analysis in terms of spectrum ratio at different distances allows elimination of many systematic error sources such as detector efficiency and theoretical antineutrino spectrum including its variations with the reactor fuel evolution. Monte-Carlo simulation is used to account for the detector energy response and for the reactor and the detector size. All the above smear the oscillation effect, yet the sensitivity remains reasonably good. The statistics, recorded in about a year of data taking with weekly cycle of detector position changes, was processed to obtain the exclusion regions [2] in the oscillations parameter space using CLs approach [3] for the most conservative estimates. Figure 3 presents the exclusion regions obtained from the DANSS data together with the allowed regions from the neutrino disappearance experiments $[4,5]$ for comparison. The parameter values, most preferred by the Reactor and Gallium Anomalies [5] (shown with a star) are excluded with the significance higher than $5 \sigma$.

DANSS collaboration is grateful to the directorates of ITEP and JINR for continuous support of this work. The collaboration appreciates the permanent assistance of the KNPP administration and Radiation Safety Department staff. The detector construction was supported by the Russian State Corporation ROSATOM (state contracts H.4x.44.90.13.1119 and H.4x.44.9B.16.1006). The operation and data analysis became possible due to the valuable support from the Russian Science Foundation grant 17-12-01145. 


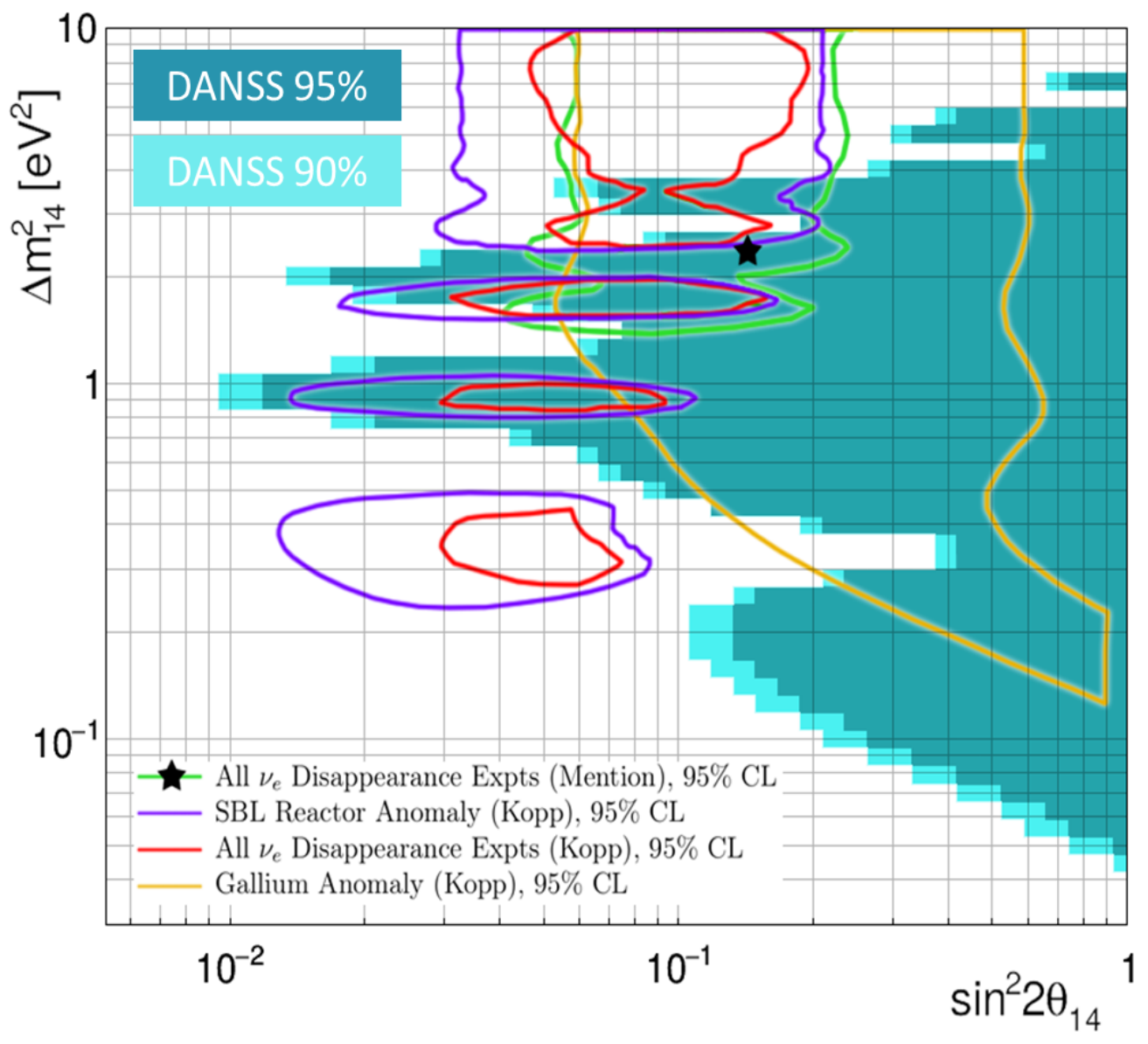

Figure 3: CLs exclusion area in the oscillation parameter space. The shaded areas represent the analysis of DANSS data. Curves show the allowed regions from neutrino disappearance experiments [4, 5], and the star is the best point from the RAA and GA fit [5].

\section{References}

[1] I. Alekseev et al., JINST 11 (11) (2016) P11011.

[2] I. Alekseev et al., Phys. Lett. B, 787 (2018) 56-63.

[3] X. Qian et al., Nucl. Inst. Meth. A 827 (2016) 63.

[4] J. Kopp, P.A.N. Machado, M. Maltoni and T. Schwetz, J. High Energy Phys. 1305 (2013) 050.

[5] G. Mention, et al. Phys. Rev. D 83 (2011) 073006. 